\title{
Pratiques
}

Linguistique, littérature, didactique

\section{Réflexions sur la conception d'un nouveau dictionnaire bilingue français-chinois d'aide à la production du FLE}

\section{Xinxia Wang}

\section{OpenEdition}

\section{Journals}

Édition électronique

URL : http://journals.openedition.org/pratiques/3503

DOI : $10.4000 /$ pratiques.3503

ISSN : 2425-2042

Éditeur

Centre de recherche sur les médiations (CREM)

\section{Édition imprimée}

Date de publication : 15 décembre 2012

Pagination : 182-194

\section{Référence électronique}

Xinxia Wang, «Réflexions sur la conception d'un nouveau dictionnaire bilingue français-chinois d'aide à la production du FLE », Pratiques [En ligne], 155-156 | 2012, mis en ligne le 18 décembre 2017, consulté le 19 avril 2019. URL : http://journals.openedition.org/pratiques/3503 ; DOI : 10.4000/ pratiques.3503 


\title{
Réflexions sur la conception d'un nouveau dictionnaire bilingue français-chinois d'aide à la production du FLE
}

\author{
WANG Xinxia
}

Beijing City University

\section{Introduction}

Traditionnellement, pour produire ou traduire des mots en langue étrangère, on consulte un dictionnaire bilingue LM-LE. Dans le cas des étudiants chinois, ce sont les dictionnaires chinois-français qui assument la fonction d'encodage. Néanmoins, les dictionnaires chinois-français seuls ont du mal à répondre aux besoins des apprenants chinois dans la production en français. Par exemple, l'apprenant qui veut exprimer 《请通知我开会时间 qǔng tōngzhī wǒ kāihuì shíjiān » trouvera l'équivalent prévenir du verbe 《通知 tōngzhī 》 dans un dictionnaire chinois-français. Mais en général, ce dernier ne fournit pas d'informations permettant d'organiser correctement la phrase basée sur ce mot. Dans ce cas, il lui faut recourir à un dictionnaire bilingue français-chinois ou un monolingue du français pour construire la phrase : «Prévenez-moi de l'heure de la réunion. ». En réalité, bien que rares soient ceux qui prennent la peine de vérifier dans un dictionnaire bilingue LE-LM ou un dictionnaire monolingue les équivalents ou les traductions trouvés dans le dictionnaire de départ (Bogaards, 2006 : 24), cette démarche est indispensable pour une expression correcte et appropriée. Afin d'insérer les équivalents trouvés dans la phrase à construire, l'apprenant ne doit pas se contenter de la forme qu'il a trouvée, il lui faut en savoir plus : sens exact, propriétés syntaxiques, combinatoires lexicales, etc.

\section{Outils de production en FLE : quels dictionnaires privilégier?}

Théoriquement, quatre types de dictionnaires bilingues sont nécessaires pour toute paire de langues : deux dictionnaires de décodage (ou de compréhension) et deux dictionnaires d'encodage (ou de production) destinés respectivement aux locuteurs de la langue source et de la langue cible. Pourtant, cette distinction fondamentale n'est pas absolue en pratique. Sur le marché lexicographique en Chine, les dictionnaires français-chinois s'adressant aux apprenants chinois assument en même temps la fonction d'encodage et celle, traditionnelle, de décodage. Imaginons la situation d'un apprenant chinois qui cherche un mot français dans un dictionnaire chinois-français pour exprimer une idée: il y trouvera une ou plusieurs formes françaises correspondant au mot chinois dont il saisit bien le sens. Or, cette démarche ne semble pas suffisante : pour s'exprimer d'une manière appropriée, il lui faut trouver la réponse à des questions qui se poseraient dans ce contexte : 
- L'équivalent français proposé par le dictionnaire correspond-il au mot chinois sur le plan sémantique et stylistique?

- Comment l'équivalent français se comporte-t-il dans le discours? Autrement dit, quelles sont ses propriétés syntaxiques et combinatoires lexicales?

Le dictionnaire chinois-français n'est pas toujours capable de répondre à ces questions. Afin de surmonter de telles difficultés qui se présentent fréquemment lors de la production, l'apprenant doit faire appel à d'autres outils susceptibles de jouer un certain rôle dans cette tâche : dictionnaire bilingue français-chinois, dictionnaire monolingue du français, voire moteurs de recherche sur Internet comme Google.fr (pour chercher des collocations adéquates, par exemple)... Parmi ces outils de production, le dictionnaire bilingue françaischinois présente le meilleur rapport qualité/investissement sur lequel l'apprenant peut compter pour trouver un maximum d'informations avec un minimum d'effort (Galisson, $1991: 26)$.

Les difficultés auxquelles sont confrontés les étudiants chinois pour produire un texte français sont assez importantes : traduction littérale de la LM, utilisation d'un vocabulaire peu varié, emploi des collocations, etc. Cependant, il n'existe pas encore un véritable dictionnaire français-chinois orienté vers l'apprentissage du français en Chine, et encore moins un dictionnaire destiné à la production.

\section{Le dictionnaire d'encodage : quelles informations privilégier?}

La production est un processus centré sur la planification sémantique concrétisé à travers l'organisation des phrases, qui, à son tour, se réalise par le moyen de la sélection lexicale. De ce fait, l'apprenant d'une langue étrangère, lorsqu'il exprime une idée dans cette langue, ne cesse d'effectuer des choix lexicaux et grammaticaux, généralement d'une manière inconsciente (Mel'čuk et Polguère, 2007 : 58). Au niveau lexical, les choix se font autour de deux axes : axe paradigmatique et axe syntagmatique. Les choix paradigmatiques consistent à identifier le(s) mot(s) juste(s) parmi toutes les possibilités sémantiques qui pourraient prendre la même place dans la phrase. Et les choix syntagmatiques, une fois le(s) mot(s) juste(s) sélectionné(s), se rapportent à leur tour à la syntaxe et à la combinatoire lexicale.

Prenons maintenant quelques exemples tirés d'exercices de nos étudiants pour expliciter les enjeux dans le processus de production :

(1) 我 特别 喜欢玩电脑。

wǒ tèbié xĭhuān wán diànnăo

moi surtout aimer jouer ordinateur

(2) 突如其来的 大

tūrúqíláide dà xuě

survenant grand neige

造成了

严重的

交通拥堵。

zàochéngle yánzhòngde jiāotōngyōngdǔ

causer grave embouteillages

Pour composer la phrase (1) en français, l'apprenant aura au moins deux choix à opérer sur le plan paradigmatique. S'il faisait appel à un dictionnaire chinois-français, il obtiendrait des traductions pour «喜欢 xìhuān » : aimer, préférer, se plaire à, avoir le goût de... et pour "玩 wán »: jouer, jouer à, s'amuser, se distraire... Les choix peuvent être faciles si l'on ne prend en compte que la dimension sémantique des unités lexicales. Or, pour formuler une phrase correcte et appropriée, on doit aussi prendre en considération d'autres éléments majeurs tels que les propriétés syntaxiques du verbe choisi. Par exemple, l'apprenant peut hésiter entre jouer l'ordinateur, jouer à l'ordinateur, jouer sur l'ordinateur, jouer avec l'ordinateur, etc.

Dans l'exemple (2), les possibilités d'équivalence (approximative) sont nombreuses sauf 
pour le mot 《雪 xuě 》 (neige). Par exemple, pour 《突如其来 (的 ) tūrúqílái(de) 》, on trouvera survenant, soudain, brusque, inattendu, imprévu... La sélection d'un «bon » mot n'est pas facile car les choix paradigmatiques et syntagmatiques se conditionnent réciproquement et ne peuvent pas se faire tout seul au cours de la production. Et les interactions qu'entretiennent les deux rendent la tâche plus complexe. En effet, tandis que les locuteurs natifs savent naturellement s'exprimer dans leur langue, « les étrangers risquent à tout bout de champ de tomber dans les pièges de la création syntagmatique » (Hausmann, 1989 : 1387). Si dans l'exemple (1), l'hésitation du choix se rapporte principalement aux propriétés syntaxiques du verbe jouer, celle du choix dans l'exemple (2) relève plutôt de la combinatoire lexicale, plus précisément des collocations : l'épithète 《大 dà » (grand) et «突 如其来 (的) tūrúqílái(de) 》 (survenant) avec sa base 《雪 xuě 》 (neige), l'épithète 《严重 (的) yánzhòng(de) 》 (grave) avec sa base 《交通拥堵 jiāotōngyōngdǔ 》 (embouteillage)

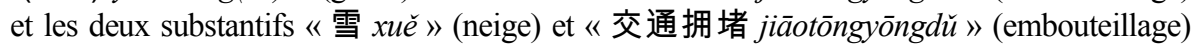
avec leur verbe support «造成 ( 了 ) zàochéng(le) 》 (causer).

Par ailleurs, on constate un autre aspect de la sélection lexicale non moins important sur l'axe syntagmatique: les actants. Les erreurs commises par les étudiants que nous enregistrons dans leurs activités productives font percevoir leur importance. Voici deux exemples concrets à fin d'illustration :

(3) *Elle mange beaucoup tous les jours et elle évolue de plus en plus grosse.

(4) *La conférence s'en tient là, vous pouvez rentrer chez vous.

Dans l'exemple (3), le verbe évoluer est mal utilisé. L'étudiant a confondu devenir qui peut avoir deux actants (le sujet et l'attribut) et évoluer qui ne porte qu'un actant (le sujet).

Dans l'exemple (4), l'étudiant s'est trompé sur la nature de l'actant de la locution verbale s'en tenir là qui ne peut prendre que l'individu comme sujet, alors que son équivalent chinois 《到此为止 dàoč́wéizhř » prend souvent « quelque chose » comme sujet.

Il résulte de cette analyse que les connaissances lexicales permettant une production correcte et appropriée résident essentiellement dans les dimensions suivantes :

- sur le plan paradigmatique : traits sémantiques de l'unité lexicale ainsi que ses relations avec d'autres unités : synonymie, antonymie, hyponymie, etc., qui servent à identifier le(s) mot(s) juste(s) ;

- sur le plan syntagmatique : propriétés syntaxiques, actancielles et combinatoires lexicales, qui servent à intégrer un mot juste dans la phrase.

Ainsi un dictionnaire d'encodage doit décrire de façon systématique les relations paradigmatiques et syntagmatiques contrôlées par les unités lexicales, plus précisément les informations sémantiques, syntaxiques, actancielles et combinatoires lexicales (surtout les collocations).

\section{Conception générale}

Afin d'élaborer un dictionnaire bilingue français-chinois qui permettrait de développer la compétence lexicale d'une façon efficace chez les étudiants chinois, surtout dans le domaine de la production, nous emprunterons à I. Mel'čuk quelques éléments de la théorie de la Lexicologie Explicative et Combinatoire (LEC) ainsi que ses adaptations théoriques et pratiques telles que le Dictionnaire Explicatif et Combinatoire (DEC) et le Lexique Actif du Français (LAF).

Grosso modo, ce nouveau dictionnaire français-chinois sera un DEC bilingue simplifié accompagné d'un index chinois-français et pourra être intitulé Dictionnaire Explicatif et Combinatoire Français-Chinois (désormais le DECFrCh). Ayant pour objectif d'aider les 
apprenants sinophones à apprendre le français et surtout à s'exprimer dans cette langue, il sera conçu selon trois principes que nous présentons ci-dessous.

\section{- Principe pratique}

À la différence du DEC qui est plutôt un ouvrage donnant la priorité à la théorie lexicologique et lexicographique, le $\mathrm{DECFrCh}$ sera un outil pratique destiné aux utilisateurs moyens. C'est-à-dire que nous ne prétendons pas donner toutes les informations à propos d'un mot individuel mais seulement celles qui peuvent être utiles à l'apprenant dans des circonstances spécifiées.

\section{- Principe pédagogique}

L'idée d'apprendre le vocabulaire en utilisant un dictionnaire bilingue est une très ancienne tradition. L'histoire des dictionnaires bilingues laisse voir une forte orientation didactique dès le début (Zöfgen, 1991 : 2894), d'autant plus que l'on connaît une époque où la spécialisation dans le domaine lexicographique, comme dans beaucoup d'autres, est de plus en plus évidente. Ainsi un dictionnaire élaboré dans une perspective pédagogique doit permettre aux apprenants une étude progressive et cohérente du lexique. Le DECFrCh est appelé donc à contenir une mine d'informations sur le lexique du français et surtout sur sa structure afin de devenir un vrai outil d'apprentissage pour les étudiants chinois : il doit constituer un moyen d'enrichir leur vocabulaire quantitativement et qualitativement. Parallèlement, il doit être un vrai support pédagogique pour l'enseignant en lui permettant de chercher l'information dont il aura besoin dans ses cours, de mettre en place des exercices d'apprentissage des connaissances lexicales et de développer la capacité des apprenants à observer et à comprendre le lexique du français (Mel'čuk et Polguère, 2007 : 54).

- Principe actif

À l'encontre des dictionnaires traditionnels français-chinois qui assument essentiellement la fonction de décodage, l'objectif principal du DECFrCh consiste à aider à l'encodage de la pensée en français. Plus précisément, il constituera un outil de production pour des apprenants chinois de tout niveau (en particulier de niveau intermédiaire et avancé) qui ont besoin d'exprimer un sens donné en organisant des phrases de façon correcte et acceptable à partir des mots. Afin de mieux établir le pont entre les concepts que l'apprenant cherche à exprimer et les ressources lexicales susceptibles de réaliser cette expression, le DECFrCh doit lui offrir le plus de moyens lexicaux nécessaires. Cette orientation implique que beaucoup d'informations lexicales qui ne sont pas indispensables dans un dictionnaire de décodage doivent faire l'objet d'une présentation systématique et explicite dans le DECFrCh. C'est que pour un apprenant d'une langue étrangère, il est généralement beaucoup plus facile de comprendre une expression idiomatique que de la produire lui-même (Mel'čuk et Polguère, 2007 : 15). Par exemple, si la présence des collocations courantes n'est pas obligatoire dans un dictionnaire de décodage, il est prioritaire d'introduire les possibilités collocationnelles dans un dictionnaire d'encodage.

De ce point de vue, le DEC ainsi que d'autres adaptations de la LEC sont pour nous un modèle idéal avec leurs descriptions lexicographiques centrées autour de trois types d'informations lexicales : sémantiques, syntaxiques et combinatoires. Ce qui constitue une présentation en profondeur des informations sur les unités lexicales ainsi que sur les relations privilégiées qu'elles entretiennent.

Bref, le DECFrCh peut être utilisé pour l'apprentissage et l'enseignement du vocabulaire français avec les renseignements qu'il fournit sur les unités lexicales: dérivations sémantiques, propriétés syntaxiques, actants, collocations, etc. D'une part, il permettra à l'apprenant d'acquérir des informations précieuses sur les mots à partir desquels il exprime un sens donné ; d'autre part, il permettra à l'enseignant de grouper aisément divers types d'informations sur le lexique français grâce auxquelles il concevra 
des activités d'apprentissage du vocabulaire et aidera ses étudiants à découvrir la structure du lexique, et ensuite le fonctionnement de la langue.

\section{Quelques notions de base du DEC}

La lexicologie explicative et combinatoire (LEC) est une branche de la théorie linguistique Sens-Texte. La description lexicale selon cette théorie se fait à l'intérieur du DEC sous forme de système cohérent et uniforme (Mel'čuk et al., 1984, Préface de Clas : XIV). Le DEC est un ouvrage théorique qui ne tient pas compte de contraintes d'ordre pratique (commerciales, typographiques, etc.). Afin de prendre en charge les deux tâches de la production - les choix paradigmatiques et les choix syntagmatiques, le DECFrCh se présentera comme un DEC pratique dont l'adaptation et la simplification s'appuient sur quelques notions de base de la LEC présentées dans ce qui suit : étiquette sémantique, dérivation sémantique, fonction lexicale, actants sémantiques et syntaxiques, schéma de régime, etc.

\section{1. Étiquette sémantique}

L'étude lexicologique et lexicographique exige des regroupements de lexies (c'est-à-dire un mot pris dans une seule acception) ayant plus ou moins une parenté sémantique. La LEC a introduit un concept important : étiquette sémantique. "Formellement, une étiquette sémantique est une lexie ou une expression française ayant une signification très générale et pouvant être utilisée comme genre prochain dans la définition lexicographique de plusieurs mots-vedettes » (Mel'čuk et Polguère, 2007 : 33).

La fonction classificatrice des étiquettes sémantiques à l'intérieur de l'ensemble des lexies est évidente. Dans le dictionnaire monolingue, l'étiquette sémantique est souvent mise en évidence par les définitions dans lesquelles elle est utilisée comme genre prochain de tous les co-hyponymes. L'examen de la définition des lexies appartenant à un même champ sémantique permet de mieux connaître leurs ressemblances et leurs dissemblances. Dans le dictionnaire bilingue qui se passe généralement de la définition, les lexicographes ont également intérêt à montrer, d'une façon ou d'une autre, ces ressemblances et dissemblances (sémantiques, syntaxiques, combinatoires, etc.).

\subsection{Dérivation sémantique}

D'après sa définition traditionnelle, la dérivation renvoie à des relations à la fois sémantiques et formelles : il s'agit de la dérivation morphologique ou famille de mots, si on utilise le terme plus courant. La notion de dérivation sémantique introduite par le LAF est plus large : « une dérivation sémantique est une relation particulière entre deux lexies : une lexie de départ et une lexie 'sémantique construite' à partir de celle-ci » (Mel'čuk et Polguère, 2007 : 22). Il est évident que cette relation est fondée uniquement sur une parenté de sens et le lien morphologique entre les lexies n'est plus une condition nécessaire. De ce fait, la dérivation au sens traditionnel n'est qu'un cas particulier de la dérivation sémantique.

\subsection{Fonction lexicale}

Dans la théorie de la LEC, la fonction lexicale (FL) est une notion importante et constitue une innovation lexicographique considérable. Il s'agit d'un outil conçu pour 
décrire toutes les relations sémantiques lexicales. Plus précisément, elle décrit une relation existant entre une lexie (appelée mot clé de la FL) et un ensemble de lexies ou d'expressions figées (appelé valeur de la FL) (Polguère, 2003 : 131).

La fonction lexicale peut être représentée par la formule suivante :

$$
\mathrm{f}(\mathrm{x})=\mathrm{y}
$$

Dans cette formule :

$$
\begin{aligned}
& \mathrm{f}=\mathrm{FL} \\
& \mathrm{x}=\text { mot clé } \\
& \mathrm{y}=\text { valeur de la FL (qui regroupe l'ensemble des lexies et des expressions qui } \\
& \text { peuvent exprimer le sens ou le rôle syntaxique donné auprès du mot clé) }
\end{aligned}
$$

Les fonctions lexicales couvrent à la fois les relations paradigmatiques (synonymes, antonymes, dérivés...) et les relations syntagmatiques (intensificateurs, verbes supports...) entre les lexies. Elle offre aux lecteurs « la totalité des moyens lexicaux nécessaires à l'expression la plus riche, la plus variée et la plus complète de la pensée et, en même temps, de garantir le choix le plus précis de la formulation appropriée » (Mel'čuk, Clas et Polguère, 1995 :129).

\subsection{Actants sémantiques et actants syntaxiques}

Pour comprendre la notion d'actant sémantique, on peut partir du verbe. Les actants d'un verbe signifient « les êtres et les choses participant au processus exprimé par le verbe » (Picoche, $1986: 47)$. Il y a des verbes à un seul actant et des verbes à deux ou trois actants. À titre d'exemple, le verbe donner contrôle trois actants sémantiques : celui qui donne, celui qui reçoit et celui qui passe de l'un à l'autre. Mais un actant peut être absent à la surface dans certaines phrases où apparaît la lexie. Dans la phrase Pierre a vendu sa voiture, les actants sémantiques désignant l'acheteur et la somme d'argent que Pierre a reçue ne sont pas exprimés syntaxiquement (Mel'čuk, Clas et Polguère, 1995 : 77-78).

De là découle la notion de l'actant syntaxique ainsi que la distinction entre l'actant syntaxique de surface, qui correspond aux compléments de type sujet et objets de la grammaire traditionnelle, et l'actant syntaxique profond, qui désigne un syntagme dépendant syntaxiquement de la lexie et exprimant un actant sémantique de celle-ci. Ce dernier ordre d'actants syntaxiques joue un rôle important dans la lexicologie et dans l'enseignement et l'apprentissage de langues étrangères car les dépendants actanciels ont une forte tendance à s'exprimer en fonction de la lexie donnée, " de façon idiomatique et capricieuse » (Mel'čuk, Clas et Polguère, 1995 : 117-118).

\subsection{Schéma de régime}

Dans le cadre du DEC, on propose pour chaque lexie prédicative une forme propositionnelle composée de la lexie-vedette et des variables désignées par des majuscules $\mathrm{X}, \mathrm{Y}, \mathrm{Z}, \mathrm{W}$... Chaque variable correspond à un actant sémantique contrôlé par la lexie en question (Mel'čuk et al., 1984 : 19).

Les actants syntaxiques profonds sont présentés de manière explicite sous forme d'un tableau appelé schéma de régime dans lequel on précise pour chaque actant syntaxique profond deux renseignements : sa forme de surface (infinitif, syntagme prépositionnel avec telle préposition, etc.) d'une part, et l'actant sémantique qui lui correspond d'autre part 
(Mel'čuk et al., 1984: 5-6). Voici le schéma de régime pour représenter la forme propositionnelle suivante ${ }^{(1)}$ :

$X$ aide $Y$ à $Z$-er de $W=\mathrm{Y}$ étant en train d'effectuer une action $\mathrm{Z}, \mathrm{X}$ emploie ses ressources $\mathrm{W}$ dans le but de causer que $\mathrm{W}$ aident $\mathrm{Y}$ à effectuer $\mathrm{Z}$

\begin{tabular}{|l|l|l|l|}
\hline $1=\mathrm{X}$ & $2=\mathrm{Y}$ & $3=\mathrm{Z}$ & $4=\mathrm{W}$ \\
\hline $1 . \mathrm{N}$ & $1 . \mathrm{N}$ & $1 . \grave{a} \mathrm{~N}$ & 1. de $\mathrm{N}$ \\
& & $2 . \grave{a} \mathrm{~V}_{\text {inf. }}$ & 2. avec $\mathrm{N}$ \\
& & 3. dans $\mathrm{N}$ & 3.par $\mathrm{N}$ \\
& & 4. pour $\mathrm{N}$ & 4. Adv \\
& & 5. pour $\mathrm{V}_{\text {inf. }}$ & \\
& & & \\
\hline
\end{tabular}

\section{Macrostructure et microstructure du DECFrCh}

La présentation que nous venons de faire sur les notions de base du DEC permet de comprendre que le rôle principal de ce genre de dictionnaires est de fournir des informations de manière systématique et exhaustive sur les unités lexicales d'une langue donnée, si bien qu'en principe, « on puisse y trouver tout ce qui est nécessaire pour construire toutes les expressions correctes de n'importe quelle pensée considérée » (Mel'čuk et al., 1984 : 4). Dans ce qui suit, nous présenterons les grandes lignes de la macrostructure et de la microstructure du dictionnaire bilingue tel que nous le concevons, dans la perspective du DEC dont on a montré l'intérêt.

Le nombre des mots-vedettes des dictionnaires français-chinois les plus consultés par les apprenants chinois varie entre $38.000^{(2)}$ et $120.000^{(3)}$. Cependant, pour arriver au million d'entrées, il faut ajouter un grand nombre de mots scientifiques et techniques qui, en général, ne sont utilisés que par les spécialistes. Selon le Français fondamental ${ }^{(4)}$, le nombre de mots utiles et effectivement employés doit être de l'ordre de 10.000 pour un individu normal (un locuteur «moyen ») s'exprimant dans sa vie courante (Picoche, 1986 : 4). Étant un outil d'aide à l'expression en français, le DECFrCh ciblera la langue générale standard contemporaine et exclura les mots scientifiques et techniques, les variétés linguistiques des classes et des groupes sociaux différents, des régions différentes, des périodes différentes... En revanche, il contiendra des lexies courantes propres à la vie quotidienne.

Ainsi, c'est l'orientation pédagogique et productive qui détermine la nomenclature du DECFrCh : elle ne contient en général qu'un nombre limité de vocables, notamment ceux qui contrôlent un ensemble significatif de lexies sur le plan paradigmatique et syntagmatique. En d'autres termes, une des vocations principales de ce dictionnaire, c'est de décrire les relations paradigmatiques et syntagmatiques contrôlées par les unités lexicales. Ce qui signifie qu'étant un DEC, bien que simplifié, le DECFrCh cherche à être exhaustif au niveau de la microstructure plutôt qu'au niveau de la macrostructure.

La microstructure du DECFrCh se composera de quatre zones d'informations :

(1) Mel’čuk, I. et al. (1984) : Dictionnaire Explicatif et Combinatoire du français contemporain, Montréal, Les Presses de l'Université de Montréal, p. 61.

(2) Xue, J.-Ch. et al. (2001) : Dictionnaire Larousse de la langue française avec explications bilingues, Pékin, Larousse/Foreign Language Teaching and Research Press.

(3) Chen, Zh.-R. (éd.) (2005) : Grand dictionnaire français-chinois du nouveau siècle, Pékin, Foreign Language Teaching and Research Press.

(4) Ministère de l'Éducation Nationale (1953), Le Français Fondamental (1 ${ }^{\text {er }}$ et $2^{\text {e }}$ Degrés), Paris, Publication de 1'Institut Pédagogique National, diffusion Sevpen. 
- zone d'introduction : transcription phonétique, partie du discours, genre pour les noms, formes féminines pour les noms et les adjectifs, renseignements sur la conjugaison des verbes irréguliers, position de l'épithète (préposé ou postposé) par rapport au nom, niveaux de langue, etc. ;

- zone sémantico-syntaxique: équivalents ou traductions, étiquette sémantique, connotation, définition en français, dérivés sémantiques (synonymes, antonymes, hyperonymes, mots de familles...), etc. ;

- zone de combinatoire lexicale: collocations et d'autres unités phraséologiques (expressions idiomatiques, phrases codées de tout genre...) ;

- zone diverse : exemples illustratifs, informations culturelles, remarques d'usage, etc.

Trois types d'informations fondamentales sur les lexies ainsi que leur fonctionnement dans le système langagier du français sont privilégiés: informations sémantiques, informations syntaxiques-actancielles et informations de combinatoire lexicale. Nous précisons ensuite quelques rubriques présentées ci-dessus qui nous semblent montrer la spécificité principale de ce dictionnaire d'encodage s'adressant aux apprenants chinois.

\subsection{Connotation}

Selon la définition du DEC, la connotation est une caractéristique quelconque que la lexie attribue à son référent et qui ne fait pas partie de la description lexicographique (Iordanskaja et Mel'čuk, $1984: 34$ ). Elle représente donc des valeurs sémantiques secondaires ajoutées à la signification de base de la lexie. Dans un dictionnaire monolingue qui vise les locuteurs natifs, il ne semble pas nécessaire d'expliciter la connotation des lexies puisque les associations d'idées relevant de la connotation sont en général partagées par l'ensemble d'une communauté linguistique. En revanche, pour un dictionnaire bilingue élaboré dans une optique contrastive, le sens connotatif est parfois indispensable à la bonne compréhension et à une réutilisation appropriée d'une lexie, surtout quand il y a des écarts entre deux lexies « équivalents » appartenant respectivement à deux systèmes langagiers. Par exemple, si la fourmi fait penser à l'avarice aux Français, cette connotation n'existe pas dans son équivalent chinois 《蚂蚁 măy $\check{l}$ 》. De plus, les connaissances connotatives facilitent la compréhension des sens figurés, des expressions métaphoriques et métonymiques transférées par une lexie donnée. La connotation constitue donc une information importante pour apprendre une langue étrangère et ouvre une bonne piste pour découvrir sa culture.

\subsection{Définition}

Dans la pratique pédagogique, on constate souvent que la compréhension d'une unité lexicale française par les apprenants chinois se fait à partir des équivalents chinois donnés par les manuels ou les dictionnaires. Ce qui constitue une importante source d'erreurs, notamment dans la production. À cet égard, la définition peut être fort utile car elle permet une maîtrise de sens bien plus précise que les équivalents, surtout quand les deux langues sont aussi distantes que le français et le chinois.

Dans le DECFrCh, la définition s'avère encore plus importante parce qu'elle offre des informations indispensables au bon usage de la lexie non seulement sur le plan sémantique, mais aussi sur le plan syntaxique et actancielle. Observons les définitions suivantes proposées à une lexie verbale et à une lexie nominale :

\section{ADMIRER vt. 赞赏，赞美；仰慕，钦佩，佩服}

Un individu ADMIRE qqn (qqch.), que (+ prop $_{\text {subj. }}$ ) pour qqch. (pour/de + inf. passé), c'est qu'il manifeste un sentiment de respect pour sa beauté ou ses qualités morales. 


\section{SOUPÇON n.m. 怀疑, 猜疑}

Le SOUPÇON d'une personne à propos de (au sujet de, envers) une autre personne sur un fait (que+ prop.), c'est une opinion ou idée qui fait attribuer à qqn des actes blâmables, mais sans preuve réelle.

Il faut dire que toutes les lexies ne possèdent pas la définition que nous venons de décrire. Ce sont plutôt les lexies prédicatives dont les actants poseraient des problèmes à la production qui seront munies d'une telle définition. Regardons maintenant deux autres définitions consacrées au substantif :

\section{VACHE n.f. 母牛，奶牛}

La VACHE est la femelle du taureau. C'est un gros animal domestique qu'on élève pour la production laitière et la viande.

\section{PARAPLUIE n.m. 雨伞 \\ Le PARAPLUIE est un instrument qui sert à protéger de la pluie.}

Il est évident que la façon de définir ces deux lexies nominales est différente de celle concernant les lexies précédentes. L'accent ici est mis plutôt sur leurs étiquettes sémantiques ou les champs sémantiques auxquels elles appartiennent ainsi que sur leurs dérivés sémantiques (pour VACHE : taureau, femelle, animal domestique, élever... ; pour PARAPLUIE : instrument, pluie, protéger). Cette différence s'explique par le fait qu'en général, les actants que contrôlent les noms concrets tels que VACHE et PARAPLUIE posent beaucoup moins de problèmes aux niveaux syntaxique et actanciel en production. Cependant, la définition donnée à des noms concrets n'est pas superflue dans notre dictionnaire: elle fournit des renseignements sémantiques supplémentaires dont l'apprenant aura besoin pour réaliser l'encodage d'une idée ou pour varier son expression par paraphrase.

\subsection{Collocations}

Les collocations constituent un des phénomènes les plus importants sur l'axe syntagmatique, plus précisément de la combinatoire lexicale. Si les collocations ne posent pas grands problèmes en compréhension, il est difficile pour un locuteur non-natif de juger de leur acceptabilité en production. En tant qu'outil d'aide à l'expression, le DECFrCh accordera une grande importance à ce type de combinatoire et fournira les collocations les plus fréquentes en recourant à certaines notions de la fonction lexicale.

Étant donné que les fonctions lexicales constituent un système fort compliqué pour les utilisateurs moyens, on ne peut pas les copier mécaniquement tel quelles. Dans un souci pédagogique, on réduira la liste des fonctions lexicales de I. Mel'čuk pour ne retenir que les plus simples et les plus utiles : intensificateur, atténuateur, verbe support, etc. (Binon, Verlinde et Selva, $2001: 56-57)$.

\subsection{Informations culturelles}

De nos jours, qui parle de la langue parle de la culture. Ce qui est encore plus pertinent pour l'apprenant parce que le développement de sa compétence communicative est indissociable de celui de sa compétence culturelle et interculturelle. C'est pourquoi nous croyons que les informations culturelles doivent occuper une place assez importante dans le DECFrCh élaboré dans une perspective pédagogique.

L'écart culturel peut s'expliquer par toute une série de situations d'équivalence partielle 
ou de non-équivalence. Quand il s'agit d'une absence d'équivalence, il est nécessaire de donner quelques précisions supplémentaires si les informations sémantiques ne sont pas suffisantes pour la compréhension de l'apprenant. Lorsqu'il s'agit d'une divergence sémantique et culturelle entre le mot français et son «équivalent» chinois, il semble indispensable de la préciser de manière à éviter des malentendus. Par exemple, pour la lexie ÉTAGE, il vaut mieux d'expliciter la différence qui existe entre les deux cultures : « 一楼 ȳ̄lóu» (traduction littérale : premier étage) signifie le rez-de-chaussée et «二楼 èrlóu » (traduction littérale : deuxième étage) signifie le premier étage en français.

Afin que le DECFrCh devienne un véritable outil d'aide à la production, deux annexes importantes seront ajoutées à la fin de la microstructure :

- un mini-dictionnaire chinois-français ou une liste de mots chinois les plus courants accompagnés de leurs équivalents français : l'objectif est de donner quelques simples pistes d'expression à l'apprenant et de l'orienter ensuite vers le texte du dictionnaire pour qu'il y trouve une riche mine de données lexicales;

- un index des étiquettes sémantiques suivis des numéros de pages concernées : grâce à sa structure hiérarchique et à son rôle dans l'accès au sens lexical, il peut servir d'un certain dictionnaire onomasiologique contribuant au processus de l'idée à exprimer aux ressources lexicales pour l'expression de cette idée.

\section{Quelques exemples illustratifs du DECFrCh}

Pour mettre en pratique les principes de la description lexicographique proposés cidessus, nous soumettons au lecteur dans ce qui suit un échantillon d'articles dont les lexiesvedettes appartiennent respectivement à la catégorie verbale, nominale et adjectivale.

REGRETTER [rəgrete] vt. 感到抱歉 ( $\rightarrow$ éprouver un sentiment négatif)

Définition

Un individu regrette qqch., de (+inf.), que (+subj.), c'est qu'il se montre désolé (auprès de qqn) de qqch. dont il est responsable.

Dérivés sémantiques

[同义词] être désolé / s'excuser 请求原谅

[同族词] regret (n.m)

Collocations

[动+副] vivement 非常抱歉

D'autres phrasèmes

Je regrette. 抱慊。对不起。请原谅。[表示抱慊或委婉的反驳]

Pouvez-vous m'indiquer la pharmacie la plus proche ? - Je regrette, je ne suis pas du quartier. 您能告诉我最近的药店在哪儿吗 ? 抱慊, 我对这儿不熟悉。

Vous êtes dans votre tort. - Non, je regrette, c'est vous qui l'êtes. 你错了。一不, 很抱慊, 错的是你。

Exemples illustratifs

Je regrette de vous avoir fait attendre. 很抱慊，让您久等了。

Remarque d'usage

无否定式。

VACHE [vaj] n.f. 母牛, 奶牛 ( $\rightarrow$ animal d'élevage)

Connotation

air stupide 蚌相; grosseur 肥胖 


\section{Définition}

La VACHE est la femelle du taureau. C'est un gros animal domestique qu'on élève pour la production laitière et la viande.

Dérivés sémantiques

[上义词] animal (n.m.) domestique 家畜

[近义词] taureau (n.m.) 公牛 / bouf (n.m.) 牛 / veau (n.m.) 牛犊

[同族词] vachette (n.f.) 小母牛 / vacherie (n.f.) 母牛棚 ; 牛奶棚 / vacher, ère

(n.) 饲养奶牛的人, 牛倌

\section{Collocations}

[名+形] laitière ( à lait) 奶牛, 乳牛

[动+名] traire les $\sim$ s 挤牛奶

D'autres phrasèmes

$\sim$ à lait〈转，俗〉摇钱树，财源 [指人]

Non, je ne te prêterai pas d'argent, je ne suis pas une vache à lait !

不，我不会借钱给你的，我不是你的摇钱树!

$\sim \mathrm{S}$ grasses 丰收，丰年; 繁荣时期，富足时期

$\sim \mathrm{S}$ maigres 饥荒，荒年; 贫困时期

Chacun son métier, les $\sim$ s seront bien gardées.

[谚]各司其职, 天下太平。

comme une $\sim$ qui regarde passer les trains

表情迟钝地，神情茫然地; 一副痴呆模样，一副蚌相

être gros comme une $\sim$ 极其肥胖

maladie de la $\sim$ folle 疯牛

montagne à $\sim \mathrm{s}$ 极易攀登的山[登山运动员用语]

plancher des $\sim \mathrm{s}$ 陆地

pleurer comme une $\sim$ (fam.) 嚎啕大哭

(parler français) comme une $\sim$ espagnole

法语讲得很獘脚, 法语讲得糟糕透顶

(pleuvoir) comme qui pisse (fam.) 下倾盆大雨

un coup (de pied) en $\sim$ (fam.)

冷不防地横踢一脚; 〈转〉出其不意的打击; 暗算, 背信奔义

queue de loc. adj. 赫色的

\section{Exemples illustratifs}

Un troupeau de $\sim \mathrm{s}$ a traversé la route. 一群奶牛穿越了公路。

J'ai marché dans une bouse de $\sim$ ! 我踩到了牛粪 !

Le soir, on rentre les pour les traire. 每天晚上, 我们把牛赶回去挤奶。

En Inde, la est un animal sacré. 在印度，母牛是一种神圣的动物。

Informations culturelles

“牛”在汉文化中是一个很受欢迎的形象，常具有褒义的内涵，如“强壮”、

“能干”、“勤劳”、“吃苦”等，“母牛”还有“慈祥、温驯”的寓意; 而在法语中， vache 的文化内涵通常是贬义的，有“春笨”、“凶狠”的象征意义。 ${ }^{(5)}$

FIER, ÈRE [fjer] adj. [作定语时置于 n. 后] 以...为荣，因...感到自豪，骄傲 $(\rightarrow$ se dit de qqn qui éprouve un sentiment positif)

(5) Dans la culture chinoise, «牛 niú » (le bœuf) représente une image bien positive et porte souvent un sens mélioratif. Par exemple, dans la symbolique chinoise « 牛 niúu » peut connoter « la force », «le savoir-faire », « l'assiduité », etc. «母 牛 múniú » (la vache) symbolise en plus « la bienveillance » et « la docilité ». En revanche, « la vache » a une connotation généralement péjorative dans la culture française et fait penser à « la stupidité » et à « la méchanceté ». 


\section{Définition}

Une personne est FIÈRE de qqch. (qqn), de (+ inf.), que (+ subj.), c'est qu'elle en est très satisfaite, très heureuse.

Dérivés sémantiques

[近义词] content, e ; heureux, se ; satisfait, e 满意的

[反义词] honteux, se 惭愧的, 差愧的 / insatisfait, e ; mécontent, e 不满的，不快的

[同族词] fierté (n.f.)

Collocations

[形+副] bien, tout, très 非常骄傲, 非常自豪

Exemples illustratifs

Elle est fière de son fils. 她为儿子感到骄傲。

Je suis fier que tu aies réussi. 我为你的成功感到光荣。

Jean vient d'avoir une fille, il en est très fier.

让刚有了一个女儿, 他神气极了。

Il n'y a pas de quoi être fier [de ton examen] !

[你的考试成绩]没什么值得骄傲的！

\section{En guise de conclusion}

Mémoriser un certain nombre de mots sans apprendre à construire des phrases n'a pas de grande valeur pratique (Wilkins, 1972 : 110). Aussi l'objectif d'apprendre le vocabulaire n'est-il pas simplement de mémoriser les formes et les sens de nouveaux mots, mais de comprendre le fonctionnement des mots dans la langue. En effet, les connaissances lexicales regroupent encore d'autres dimensions: comportement syntaxique dans un syntagme ou dans une phrase; sens référentiel (polysémie et usages métaphoriques éventuels), affectif (ensemble des connotations), pragmatique (adéquation en situation) ; relations lexicales de la lexie avec d'autres lexies : synonymie, antonymie, hyponymie, etc.; collocations les plus fréquentes (Laufer, 1994: 99). Bref, apprendre une unité lexicale, c'est surtout maîtriser ses propriétés sémantiques, syntaxiques et combinatoires, qui constituent les informations essentielles pour la compréhension et en particulier pour la production. C'est la raison pour laquelle ces trois catégories d'informations forment le contenu principal que le $\mathrm{DECFrCh}$ cherche à décrire. Il nous semble que cette particularité qui met l'accent sur les rapports paradigmatiques et syntagmatiques des lexies pourra garantir plus de succès au sinophone producteur de français que la description lexicographique traditionnelle faite dans les dictionnaires français-chinois existants.

S'inscrivant dans le cadre de l'enseignement et de l'apprentissage du vocabulaire du FLE, notamment sur le plan productif, le DECFrCh aura deux objectifs : d'une part, il aidera les étudiants à optimiser leur apprentissage du vocabulaire ; d'autre part, il permettra à l'enseignant d'explorer la possibilité d'une meilleure approche du lexique en classe de FLE. C'est-à-dire qu'au-delà de la dimension d'apprentissage, le DECFrCh aura pour but de fournir une ressource pédagogique aux enseignants, en particulier une gamme de modes d'exploitation des relations paradigmatiques et syntagmatiques entre les éléments du lexique. En prenant le DECFrCh comme point de départ, l'enseignant pourra mettre en place de nombreuses activités pédagogiques pour développer les connaissances lexicales des apprenants ainsi que leur capacité à observer et à comprendre la structuration et le fonctionnement du lexique français. Les utilisations potentielles d'un tel dictionnaire dans l'enseignement du vocabulaire pourront être considérables. De plus, les informations qu'il propose de réunir pourront servir de support à une exploration plus approfondie de notions clés tant en lexicologie qu'en didactique du vocabulaire. 


\section{Références}

BinON, J., Verlinde, S. \& SElVA, T. (2001): «Lexicographie pédagogique et enseignement/apprentissage du vocabulaire en français langue étrangère ou seconde (FLES) : un mariage parfait », Cahiers de lexicologie, $\mathrm{n}^{\circ} 78,2001 / 01$, pp. 41-63.

BogaARDs, P. (2006) : " Produire en L2 au moyen d'un dictionnaire bilingue », Szende, T. (éd.) : Le français dans les dictionnaires bilingues, Paris, Éditions Champion.

Galisson, R. (1991): De la langue à la culture par les mots, coll. «Didactique des langues étrangères "), Paris, CLE International.

HAUSMANN, F. J. (1989) : «Les dictionnaires pour l'enseignement de la langue étrangère : français », Hausmann, F. J., Reichmann, O., Wiegand, H. E. \& Zgusta, L. (éds.) : Dictionnaires. Encyclopédie internationale de lexicographie, Berlin/New York, Walter de Gruyter, tome 2, pp. 1386-1390.

IORDANSKAJA, L. \& MEL'ČUK, I. (1984): «Connotation en sémantique et lexicographique », Mel'čuk I. et al. (1984) : Dictionnaire Explicatif et Combinatoire du français contemporain, Montréal, Les Presses de l'Université de Montréal.

IORDANSKAJA, L. \& MEL'ČUK, I. (1997) : « Le corps humain en russe et en français, vers un dictionnaire explicatif et combinatoire bilingue $»$, Cahiers de lexicologie, $\mathrm{n}^{\circ} 70$, 1997/01, pp. 103-135.

LAUFER, B. (1994) : «Appropriation du vocabulaire : mots faciles, mots difficiles, mots impossibles », Singleton, O. (éd.) : "L'acquisition du lexique d'une langue étrangère ", AILE, n³ - 1994, pp. 97-113.

MEL'ČUK, I. et al. (1984, 1988, 1992, 1999) : Dictionnaire Explicatif et Combinatoire du français contemporain, Montréal, Les Presses de l'Université de Montréal.

Mel'ČUK, I., Clas, A. \& POLGUÈRE, A. (1995) : Introduction à la lexicologie explicative et combinatoire, Louvain-la-Neuve (Belgique), Duculot.

MEL'ČUK, I. \& POLGUÈRE, A. (2007) : Lexique actif du français : l'apprentissage du vocabulaire fondé sur 20000 dérivations sémantiques et collocations du français, Bruxelles, De Boeck.

Picoche, J. (1986) : Structures sémantiques du lexique français, Paris, Éditions Fernand Nathan.

POlguÈre, A. (2003) : Lexicologie et sémantique lexicale : notions fondamentales, coll. « Paramètres (Montréal) », Montréal, Les Presses de l'Université de Montréal.

SzENDE, T. (1999a) : « Problems of exemplification in bilingual dictionaries », Dolezal, F. F. M., Rey, A., Roelcke, T., Wiegand, H. E., Wolski, W. \& Zgusta, L. (éds.) : Lexicographica, 15/1999, TüBingen, Niemeyer, pp. 198-228.

WILKINS, D. (1972) : Linguistics in language teaching, London, Arnold.

ZÖFGEN, E. (1991) : « Bilingual learner's dictionaries », Hausmann, F. J., Reichmann, O., Wiegand, H. E. \& Zgusta, L. (éds.) : Dictionnaires. Encyclopédie internationale de lexicographie, Berlin/New York, Walter de Gruyter, tome 3, pp. 2888-2903. 